\title{
An Audit of Use of Supraglottic Airway Devices in Pediatric Patients
}

\author{
${ }^{1}$ Neha Dargad, ${ }^{2}$ Nandini Dave
}

\section{ABSTRACT}

Aim: Pediatric patients have unique anatomical, physiological and pharmacological characteristics. The process of administering anesthesia for pediatric surgeries is quite challenging. Such cases are usually performed under general anesthesia using face masks, endotracheal tubes (ETT) or supraglottic airways (SGA) depending upon type and duration of surgery. Use of SGA has various advantages over the other two and their use is increasing day by day. We carried out an audit retrospectively to extract data of surgeries where SGA were used over a duration of six months. Primary objective was to delineate percentage of usage of SGA and secondary were to study associated complications and identify areas of improvement, if any.

Materials and methods: Subsequent to International Electrotechnical Commission (IEC) approval, all perioperative details related to patients and surgeries were collected from anesthesia records. A number of other parameters were also recorded.

Results: Number of patients managed under SGA during 6 months duration were 120 as compared to total of 400 . Thus, the usage was $30 \%$. There was no difficult SGA placement. Neuromuscular blockers were used in 10\% cases. Dislodgement of device was noted in $12.5 \%$ patients and laryngospasm in $10 \%$. Change of size of device was required in seven patients weighing $10 \mathrm{~kg}$.

Conclusion: The practice of use of these devices has revolutionized the field of pediatric anesthesia with advantages like avoidance of use of muscle relaxant. They are very tachydidactic and freindly to use. Some vigilance is required to prevent and treat complications associated with their use.

Clinical siginficance: The implications of SGAs are becoming wider day by day and in near future with more advance devices, they might still have wider applications than endotracheal tubes.

Keywords: Bronchospasm, Dislodgement, Laryngospasm, Pediatric.

How to cite this article: Dargad N, Dave N. An Audit of Use of Supraglottic Airway Devices in Pediatric Patients. Res Inno in Anesth 2018;3(2):33-36.

${ }^{1}$ Medical Consultant, ${ }^{2}$ Additional Professor

${ }^{1}$ Hinduhrudaysamrat Balasaheb Thackarey Medical College and Dr Rustom Narsi Cooper Municipal General Hospital, Mumbai, Maharashtra, India

${ }^{2}$ Seth Gordhandas Sunderdas Medical College and King Edward Memorial Hospital, Mumbai, Maharashtra, India

Corresponding Author: Neha Dargad, Medical Consultant, Hinduhrudaysamrat Balasaheb Thackarey Medical College and Dr Rustom Narsi Cooper Municipal General Hospital, Mumbai, Maharashtra, India, e-mail: kasat.neha21@gmail.com

\section{Source of support: Nil}

Conflict of interest: None

\section{INTRODUCTION}

Pediatric patients are not just small adults. They have unique anatomical, physiological and pharmacological characteristics. The process of administering anesthesia for pediatric surgeries is quite challenging. In our institute number of such cases are usually performed under general anesthesia using face masks, ETT or SGA depending upon the type and duration of surgery. Use of SGA has various advantages over the other two, and their use is increasing day-by-day.

Advantages of SGA over face mask:

- Provides more secure and reliable means of ventilation

- Hands of anesthetist are free

- Can be easily inserted by clinical/nonclinical staff as it requires minimal training

- Lower risk of aspiration and less operating room pollution.

Advantages of SGA over ETT are:

- Avoids need for laryngoscopy and associated stress response

- Avoids need of muscle relaxants

- Reduced requirement of anesthetic agents for airway insertion

- Provides effective ventilation similar to ETT

- Easier to insert and short learning curve

- Lower incidence of postoperative sore throat as compared to ETT.

We carried out an audit retrospectively to extract data of 6 months duration of surgeries where SGA was used. The primary objective was to delineate the percentage of usage of SGA and secondary were to study associated complications and identify areas of improvement if any.

\section{MATERIALS AND METHODS}

Subsequent to IEC approval, all perioperative details related to patients and surgeries for 6 months were collected from anesthesia records.

\section{Inclusion Criterion}

- All elective surgeries where SGA was used

- Pediatric patients less than 12 years of age

- ASA grades 1 and 2 patients. 


\section{Exclusion Criterion}

- Surgeries where alternative devices were used for airway management

- Emergency surgeries

\section{Outcome Variables}

The parameters surveyed were:

- Type of SGA (I-gel, Proseal LMA, Classic) used

- Age and weight of the patient

- Number of attempts required to insert SGA

- Type and duration of surgery

- Position of patient

- The patient maintained on spontaneous or controlled ventilation

- Associated complications

- Postoperative complaints (sore throat, hoarseness)

- Time to discharge

A standard proforma for recording these parameters was used. SGA was inserted by anesthesia trainees and by consultants only if the first attempt failed. Successful device placement and adequate ventilation were evidenced by bilateral chest excursion, squarewave capnogram tracing with positive pressure ventilation and the absence of airway obstruction. Difficult SGA placement was defined as more than three attempts taken to insert SGA. Desaturation was defined as a fall in saturation to less than $92 \%$ and hypercarbia as a rise in end-tidal $\mathrm{CO}_{2}$ more than $50 \mathrm{~mm} \mathrm{Hg}$.

\section{Power Calculation and Statistical Analysis}

This was a purely observational study with no power calculations. For extracting a percentage of usage of SGA, a total number of surgeries performed during the same period was taken as the denominator. Data were assessed for normality and presented as mean (SD), median, range, and frequency (percentage).

\section{RESULTS}

A number of patients managed under SGA during 6 months duration were 120 as compared to a total number of 400 . Thus, the usage was $30 \%$.

Table 1: Patient demographics

\begin{tabular}{lll}
\hline Demographic & Variable & $n=120$ \\
\hline Gender (n, \%) & $\mathrm{F}$ & $17(14.1 \%)$ \\
& $\mathrm{M}$ & $103(85.83 \%)$ \\
Age (years) & Mean & 4.67 \\
& Range & $0.5-13$ \\
& SD & 3.3628 \\
Weight $(\mathrm{kg})$ & Mean & 14.89 \\
& Range & $3-45$ \\
& SD & 7.26 \\
\hline
\end{tabular}

Among 120 patients, $14.1 \%$ were females, and $85.83 \%$ were males. The mean age was 4.6708 years (Table 1 ).

I-gel was used SGA in $91 \%$ of cases, proseal in $28 \%$ and classic SGA was used in just one case. Ninety-five percent of SGA were inserted in the first attempt by anesthesia trainees. Four patients required the second attempt, and only two cases required the third attempt. There was no difficult SGA placement. Nearly, $72.5 \%$ of cases were maintained on controlled ventilation whereas spontaneous ventilation was used in $27.5 \%$ cases. Neuromuscular blockers were used in $10 \%$ of cases (Table 2).

Dislodgement of the device was noted in $12.5 \%$ of patients. On the other hand, laryngospasm was observed in $10 \%$ of patients. Change of the size of the device was required in seven patients weighing 10 kilograms. Desaturation, aspiration, hypercarbia was not seen in any patient. Trauma defined as the presence of blood on the device was also absent (Table 3).

The mean duration of surgery was 1.4687 hours. The mean duration for days till discharge was 3.4833.

In $63.3 \%$ cases, patients were positioned supine for surgery and $35 \%$ in lithotomy. Hypospadias is repaired accounted for $28.3 \%$ followed by cystoscopy (30\%).

Table 2: Type of SGA, no. of attempts to insert SGA and type of ventilation

\begin{tabular}{lll}
\hline Type of SGA used & I-gel & $91(75.83 \%)$ \\
& Proseal LMA & $28(23.33 \%)$ \\
& Classic LMA & $1(0.83 \%)$ \\
\hline No. of attempts & 1st & $114(95 \%)$ \\
& $2 n d$ & $4(3.33 \%)$ \\
& 3rd & $2(1.66 \%)$ \\
\hline Type of ventilation & Controlled & $87(72.5 \%)$ \\
& Spontaneous & $33(27.5 \%)$ \\
\hline
\end{tabular}

Table 3: Complications associated with SGA

\begin{tabular}{|c|c|c|c|}
\hline Complication & $n=120$ & $\begin{array}{l}\text { No. of } \\
\text { patients }\end{array}$ & $\begin{array}{l}\text { Percentage } \\
\text { (\%) }\end{array}$ \\
\hline Dislodgement of SGA & & 15 & 12.5 \\
\hline \multirow{2}{*}{$\begin{array}{l}\text { Laryngospasm/ } \\
\text { bronchospasm }\end{array}$} & Induction & 1 & - \\
\hline & Emergence & 11 & 10 \\
\hline Desaturation & & 0 & 0 \\
\hline Aspiration & & 0 & 0 \\
\hline Hypercarbia & & 0 & 0 \\
\hline Trauma & & 0 & - \\
\hline \multirow[t]{3}{*}{ Alternative device used } & $\begin{array}{l}\text { Different } \\
\text { size SGA } \\
\text { used }\end{array}$ & 5 & 10 \\
\hline & Face mask & 4 & \\
\hline & ETT & 3 & \\
\hline $\begin{array}{l}\text { Sore throat/change of } \\
\text { voice }\end{array}$ & & 0 & 0 \\
\hline
\end{tabular}


Table 4: Position for surgery and type of surgery. Data expressed as a percentage

\begin{tabular}{lll}
\hline \multirow{2}{*}{$\begin{array}{ll}\text { Position for } \\
\text { surgery }\end{array}$} & Supine & $76(63.3 \%)$ \\
& Lithotomy & $42(35 \%)$ \\
& Lateral & $02(1.66 \%)$ \\
\hline \multirow{3}{*}{ Type of surgery } & Hypospadiasis repair & $34(28.3 \%)$ \\
& Cystoscopy & $36(30 \%)$ \\
& Others & $50(41.6 \%)$ \\
\hline
\end{tabular}

Remaining surgeries were open herniotomy, open orchidopexy, lymph node biopsy, etc., (Table 4).

\section{DISCUSSION}

The SGA is a device that facilitates oxygenation and ventilation while sitting immediately outside the larynx to form a perilaryngeal seal. They are an established part of the routine, emergency pediatric airway management, and neonatal resuscitation. The first SGA was invented by Dr Archie Brain in 1988, ${ }^{1}$ since then over the past 30 years many variations and many new SGAs have come into practice.

Early trials found that the design of pediatric LMA was a similar version of the adult and not anatomically designed for children. Since then, improvements in the design and availability of sizes [size $1(0-5 \mathrm{~kg})$ to size 3 $(30-50 \mathrm{~kg})]$, together with favorable clinical experience have led to the increasing use of SGA.

The classic, proseal, and I-gel sizes 1, 1.5, 2, 2.5 and 3 are suitable for children of various ages. Fastrach and CTrach are not available in pediatric sizes. The size of a device suitable for a child is decided by his weight. The reference range is written on the LMA tube close to the distal end along with the cuff volume to be used.

We did a retrospective audit of 6 months of data to know the trends in our practice of using SGA in the pediatric age group. The percentage of usage of SGA was $30 \%$. Out of 400 surgeries undertaken in 6 months period, 120 were performed under SGA, 80 cases under face mask and 200 cases under ETT. The usage of ETT was 50\%. Thus, over the years SGA has been used for a variety of cases where ETT were used earlier.

Out of $120,14.1 \%$ were females and $85.83 \%$ males, urogenital issues being more common in males.

I-gel was used in most patients in view of ease of insertion, availability, and presence of gastric drain. Most SGAs were inserted in the first attempt. Only 3\% required a second attempt and $1.66 \%$ third attempt. There was no difficult SGA placement. Most SGAs were placed by anesthesia trainees with little experience with the device. Thus, proving that their learning curve is very low and are quite easy to insert. ${ }^{2}$ The ones requiring second and third attempts were handled by consultants.

Patients were maintained on controlled ventilation in $72.5 \%$ and on spontaneous in $27.5 \%$ subject. Muscle relaxants were required in 12 cases. Thus, demonstrating that SGAs can be efficiently used without the need of a paralyzing agent.

Airway obstruction occurs due to malpositioning, folding of the epiglottis, biting on the tube, laryngospasm. Lingual edema following extubation can lead to difficult airway situation. Trauma to lips, gingiva, teeth, tongue can occur because of inappropriate size. Aspiration of stomach contents is a potential hazard, as they do not form an airtight seal around the larynx. Limiting the use to fasted patients and preventing gastric distention can avoid this problem.

The smaller the child, the higher the risk of complications. ${ }^{3,4}$ Most problems have been reported with the use of the size one SGA. Our audit showed similar results. Change of airway device was documented in $10 \%$. Three cases required a shift to ETT out of which, two were posted for cystoscopy with lithotomy position. So, change in the position must have caused dislodgement of SGA. Jagannathan et al. did a study comparing I-gel with Supreme LMA size $1.5,2,2.5$ and 3 no. and concluded that Igel required a greater number of manipulations to maintain patent airway. ${ }^{5}$

We found that in borderline weight (example $10 \mathrm{~kg}$ ) when 1.5 I-gel was used, it had to be replaced by 2 no. I-gel because of a leak or inefficient ventilation. So, we recommend the use of bigger size whenever such overlapping of weights is seen. Seven cases were noted which required a change of the size of the device. Abukawa et al. did a study in 70 children with I-gel 1, 1.5 and 2 and they concluded that complication rates were higher with 1.5 number I-gel. ${ }^{6}$

Dislodgement of the device was seen $12.5 \%$. It was mainly seen when the patient's position was changed to ensure a clear airway, considerable vigilance is required when fixing I-gel in the mouth and to avoid the negative effects of flexion of the proximal tubing. ${ }^{7}$ Careful positioning, fixation, and handling of the device is the key to this problem.

Laryngospasm occurred in $10 \%$ patients, in one patient at induction while in remaining at the time of removal of the device. The SGA should not be removed in light planes as this may cause coughing, laryngospasm, hypersalivation or desaturation. 8,9 We recommend removal in a deeper plane of anesthesia when the patient is not wide awake but breathing spontaneously. In most cases, CPAP helped in relieving spasm. Three cases were treated with injection propofol $1 \mathrm{mg} / \mathrm{kg}$ and two with succinylcholine $0.5 \mathrm{mg} / \mathrm{kg}$ to break laryngospasm.

Hypercarbia is mainly associated with spontaneous ventilation. Spontaneous ventilation (increase work of 
breathing, low FRC) and use of closed circuit all amount to hypercarbia. Most patients were maintained on controlled ventilation with inhalational agent and propofol, thus explaining the absence of hypercarbia. Shorter duration of surgeries can relate to the absence of airway edema, sore throat, and hoarseness in our audit.

About $29.1 \%$ of patients got discharged on the same day and $28.3 \%$ on a subsequent day. In our practice, social reasons like staying at long distance, lack of immediate access to medical help prevent early discharge.

There were few limitations like the data being collected retrospectively which may have resulted in underreporting. ENT surgeries were not included. Airway complications would have been possibly more frequent in that case.

\section{CONCLUSION}

The overall usage of SGA in pediatric patients was found to be $30 \%$. The practice of use of these devices has revolutionized the field of pediatric anesthesia. They have a number of advantages including avoidance of the use of muscle relaxant. The SGAs are very tachydidactic and friendly to use. Some vigilance is required to prevent and treat complications associated with their use. Their implications are becoming wider day by day and in the near future with more advanced SGAs, they might still have wider applications than endotracheal tubes.

\section{REFERENCES}

1. Brain AI. The development of the Laryngeal Mask-a brief history of the invention, early clinical studies and experimental work from which the Laryngeal Mask evolved. Eur J Anaesthesiol 1991;4(Supp 1):5-17.

2. Beringer RM, Kelly F, Cook TM, et al. A cohort evaluation of the paediatric i-gel ${ }^{\mathrm{TM}}$ airway during anaesthesia in 120 children. Journal of the Association of the Anaesthetist of Great Britain and Ireland 2011;66:1121-1126.

3. Mason DG, Bingham RM. The laryngeal mask airway in children. Anaesthesia 1990;45:760-763.

4. Lopez-Gil M, Brimacombe J, Alvarez M. Safety and efficacy of the laryngeal mask airway: A prospective survey of 1400 children. Anaesthesia 1996;51:969-972.

5. Jagannathan N, Sommers K, Sohn LE, et al. A randomized equivalence trial comparing the I-gel and laryngeal mask airway Supreme in children. Pediatr Anesth 2013;23: 127-133.

6. Abukawa Y, Hiroki K, Ozaki M. Initial experience of the i-gel supraglottic airway by the residents in pediatric patients. J Anesth 2012;26:357-361.

7. Hughes C, Place K, Berg S, et al. A clinical evaluation of the i-gel ${ }^{(\mathrm{TM})}$ supraglottic airway device in children. Pediatr Anesth 2012;22:765-771.

8. Kitching AJ, Walpole AR, Blogg CE. Removal of the laryngeal mask airway in children: anaesthetized compared with awake. Br J Anaesth 1996;76:874-876.

9. Lee J, Kim J, Kim S, Kim C, et al. Removal of the laryngeal tube in children: anaesthetized compared with awake. Br J Anaesth 2007;98:802-805. 